\title{
Keratosis reduces sensitivity of anal cytology in detecting anal intraepithelial neoplasia
}

\author{
Adam C. ElNaggar ${ }^{\mathrm{a}, *}$, Joseph T. Santoso ${ }^{\mathrm{b}}$, Huiwen Bill Xie ${ }^{\mathrm{c}}$ \\ a Department of Obstetrics \& Gynecology, University of Tennessee Health Science Center, Memphis, TN, USA \\ ${ }^{b}$ Division of Gynecologic Oncology, University of Tennessee-West Clinic, Memphis, TN, USA \\ c Pathology Group of the MidSouth, Trumbull Laboratories, Memphis, TN, USA
}

\section{A R T I C L E I N F O}

\section{Article history:}

Received 9 August 2011

Acceptance 12 October 2011

Available online 20 October 2011

\section{Keywords:}

Cytology

Anal Intraepithelial neoplasia

Pathology

Screening

Anoscopy

\begin{abstract}
A B S T R A C T
Objective. To identify factors that may contribute to poor sensitivity of anal cytology in contrast to the sensitivity of anoscopy in heterosexual women.

Methods. We analyzed 324 patients with biopsy confirmed diagnosis of genital intraepithelial neoplasia (either vulva, vaginal, or cervical) from 2006 to 2011 who underwent both anal cytology and anoscopy. Cytology, anoscopy, and biopsy results were recorded. Biopsy specimens underwent independent analysis for quality of specimen. Also, biopsy specimens were analyzed for characteristics that may contribute to correlation, or lack thereof, between anal cytology and anoscopic directed biopsy.

Results. 133 (41\%) patients had abnormal anoscopy and underwent directed biopsy. 120 patients with normal anal cytology had anoscopy directed biopsies, resulting in 58 cases of AIN (sensitivity 9.4\%; 0.039-0.199). This cohort was noted to have extensive keratosis covering the entire dysplastic anal lesion. 18 patients yielded abnormal anal cytology. Of these patients, 13 had anoscopic directed biopsies revealing 6 with AIN and absent keratosis (specificity 88.6\%; 0.78-0.95). The $\kappa$ statistic for anal cytology and anoscopy was $-0.0213(95 \% \mathrm{CI}=$ $-0.128-0.086$ ).

Conclusion. Keratosis reduces the sensitivity of anal cytology. Furthermore, anal cytology poorly correlates with anoscopy in the detection of AIN ( $\kappa$ statistic $=-0.0213$ ).
\end{abstract}

(c) 2011 Elsevier Inc. All rights reserved.

\section{Introduction}

The National Cancer Institute estimates that 5,260 new cases of anal cancer were made in 2010 . This constitutes a near doubling in the anal cancer rate over the past 10 years. The recently released Surveillance Epidemiology and End Results (SEER) report cites a statistically significant rise in the annual percentage change (APC) of 2.4 from 1992 to 2008 [1].

Conversely, cervical cancer APC over the same time period is -2.7 , largely attributable to improved screening modalities and guidelines [1]. Routine Pap smear screening has reduced the incidence of cervical cancer from 40 per 100,000 to 8 per 100,000. Because of this success, some have advocated anal intraepithelial neoplasia (AIN) screening using anal cytology at least in certain high risk population [2,3]. Risk factors for developing anal cancer, in both men and women, include anogenital human papillomavirus infection, anal receptive intercourse, multiple sexual partners, history of sexually transmitted disease, immunosuppression, and history of anal condyloma. Women with anal cancer

\footnotetext{
* Corresponding author at: Dept. of Obstetrics \& Gynecology, Suite E102, 853 Jefferson Ave, Memphis, TN 38163, USA. Fax: + 19014484701.

E-mail address: aelnagga@uthsc.edu (A.C. ElNaggar).
}

are likely to have a prior history of cervical intraepithelial neoplasia or cervical carcinoma $[4,5]$.

Palefsky reported the sensitivity of anal cytology for detection of biopsy-proven AIN to be $69 \%$ in HIV-positive and $47 \%$ in HIV-negative men [6]. However, our study and others suggest that anal cytology is not a sensitive method for screening for AIN [5]. In our previous study, we evaluated 205 patients with genital dysplasia for both anal cytology and colposcopy. Anal cytology had a sensitivity of $8 \%$ and specificity of $94 \%$ in the diagnosis of AIN when compared to biopsy. Lee et al had similarly poor sensitivity in their study of 169 MSM, noting 90 (53\%) of biopsy proven anal dysplasia had normal anal cytology [7].

It has been hypothesized that poor cellularity and excess contaminants may be the source of reduced sensitivity in anal cytology [8]. The focus of this study is to identify factors that may contribute to poor sensitivity of anal cytology in contrast to the sensitivity of anoscopy in heterosexual women.

\section{Methods}

The study consisted of a 339 consecutive patients with genital (vulvar, vaginal or cervical) intraepithelial neoplasia referred to the University of Tennessee-West Clinic gynecologic oncology division from June 2006 to December 2010. 
The study was approved by our University Hospital's Institutional Review Board. The sample size was estimated based on heterosexual American women. The prevalence of AIN in the study population was assumed to be around $10 \%$. This prevalence was estimated with a $95 \%$ confidence interval with a precision of $5 \%$ on each side of the estimate. A probability of $80 \%$ was desired to have the confidence interval with such precision. Using the SAS POWER procedure (SAS Institute, Cary, NC), the required sample size was found to be a minimum of 180 participants. Patients were included in the study if they had a confirmed diagnosis of genital intraepithelial neoplasia (using biopsies of the vulva, vagina or cervix). Patients were excluded if they had a history of any anogenital cancer (cervix, vulva, vagina, or anus) or absence of a rectum. All patients were evaluated as part of our clinical practice with anal cytology and anoscopy by one gynecologic oncologist who was trained in high-resolution anoscopy. Medical history and demographic data such as age, and sexual history were also collected from the chart. Any unique identifiers were deleted to protect patients' privacy. Anonymous data were reviewed in detail.

Vulvar, vaginal, and cervical examinations were done in the usual dorsolithomy position. 3\% acetic acid was applied and the patients were carefully evaluated with colposcopy. Then, an anal smear was obtained using a plastic cotton swab, soaked in normal saline, then gently inserted until resistance from the wall of the rectum was met (approximately $4.5 \mathrm{~cm}$ ). The swab was withdrawn with lateral pressure, using a spiral motion, in order to sample the entire circumference of the anal canal. The swab was processed using the ThinPrep ${ }^{\mathrm{TM}}$ (Hologic, Inc. Marlborough, MA) technique. Next, high-resolution anoscopic exam was performed by inserting a plastic disposable anoscope lubricated with water into the anus and $3 \%$ acetic acid was sprayed into the mucosa [9]. A cotton swab soaked in 3\% acetic acid was then applied evenly to the deeper anal canal prior to visualization. Anoscopic examination with $16 \times$ magnification was performed. Any abnormalities (acetowhite changes, raised lesions, discolorations) were biopsied. A local anesthetic (Lidocaine 1\% without epinephrine) was injected before biopsy of lesions distal to the dentate line. Digital vaginal and rectal examinations were also performed after colposcopy and anoscopy.

All of the anal cytology slides were reviewed for adequacy of the specimen based on the Bethesda 2001 criteria: minimum cellularity of 1-2 nucleated squamous cells per high power field (hpf) for ThinPrep specimens. Obscuring factors such as fecal material, bacteria, and inflammation may hinder evaluation, and when the majority of nucleate squamous cells are obscured, specimens would be considered unsatisfactory for evaluation [8].

Results were recorded and Cohen's kappa coefficient ( $\kappa$ ) was used to assess agreement.

\section{Results}

339 patients were identified (Fig. 1). 15 patients declined anal examination and were excluded. All participants were heterosexual women with an average age of 39 (range 14 to 83). During the study, 35\% were currently sexually active. 30 (9.2\%) patients were immunocompromised (16 HIV, 9 autoimmune disorders, 5 on suppression for organ transplantation).

The remaining 324 patients underwent anal cytology and high resolution anoscopy. 134 (41.4\%) patients had abnormal anoscopy and underwent directed biopsy, with the exception of one patient who declined. Anoscopic directed biopsy yielded 64 patients with AIN (35 AIN I, 6 AIN II, 20 AIN III) and three with non-AIN abnormalities (candida, lichen sclerosis, and lichen simplex). 120 patients with normal anal cytology had anoscopy directed biopsies, resulting in 58 cases of AIN (sensitivity 9.4\%; 0.039-0.199). Out of 324 patients, there were 18 patients with abnormal anal cytology (13 ASCUS, 4 LSIL, 1 ASC-H). Of these patients, 13 had anoscopic directed biopsy revealing 6 with AIN (3 AIN I, 1 AIN II, 2 AIN III) and 7 patients with normal anal biopsy results (specificity $88.6 \%$; 0.78-0.95). The false positive rate (FPR) of anal cytology is $57 \%(0.296-0.812)$. The $\kappa$ statistic was $-0.0213(95 \% \mathrm{CI}=-0.128-0.086)$, indicating poor agreement between anal cytology and biopsy results.

Independent analysis of previously submitted pathology biopsy slides revealed the cohort of 58 patients with negative anal cytology and biopsy proven AIN to have extensive keratosis (Fig. 2) covering the entire dysplastic anal lesion. Of the 18 cohort patients with abnormal cytology, 6 patients had AIN without keratosis covering the epithelial layer (Fig. 3). Anal cytology correlation to anal dysplasia is shown in Fig. 4.

\section{Discussion}

The inverse relationship between presence of keratosis and correlation between cytology and biopsy suggest that keratosis may contribute to the poor sensitivity of anal cytology in detecting AIN. When compared

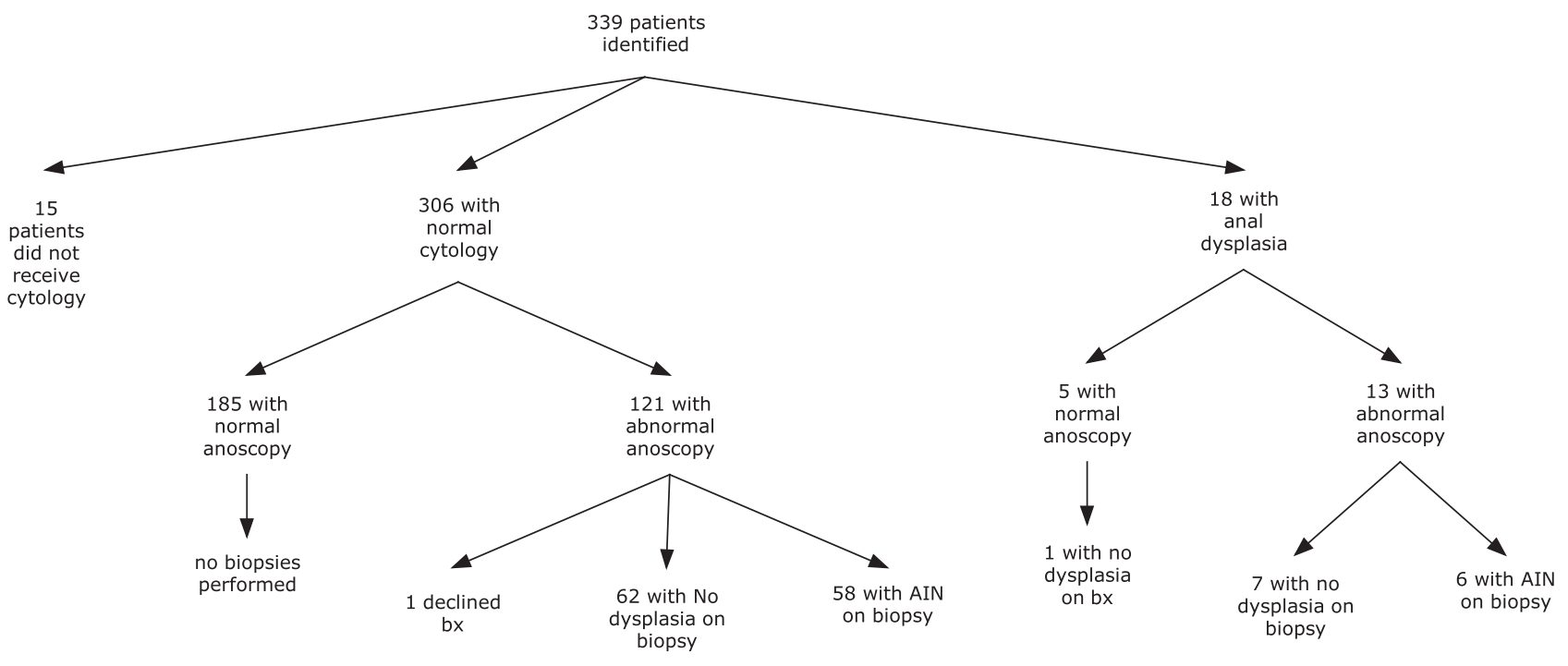

Fig. 1. Patient distribution. 


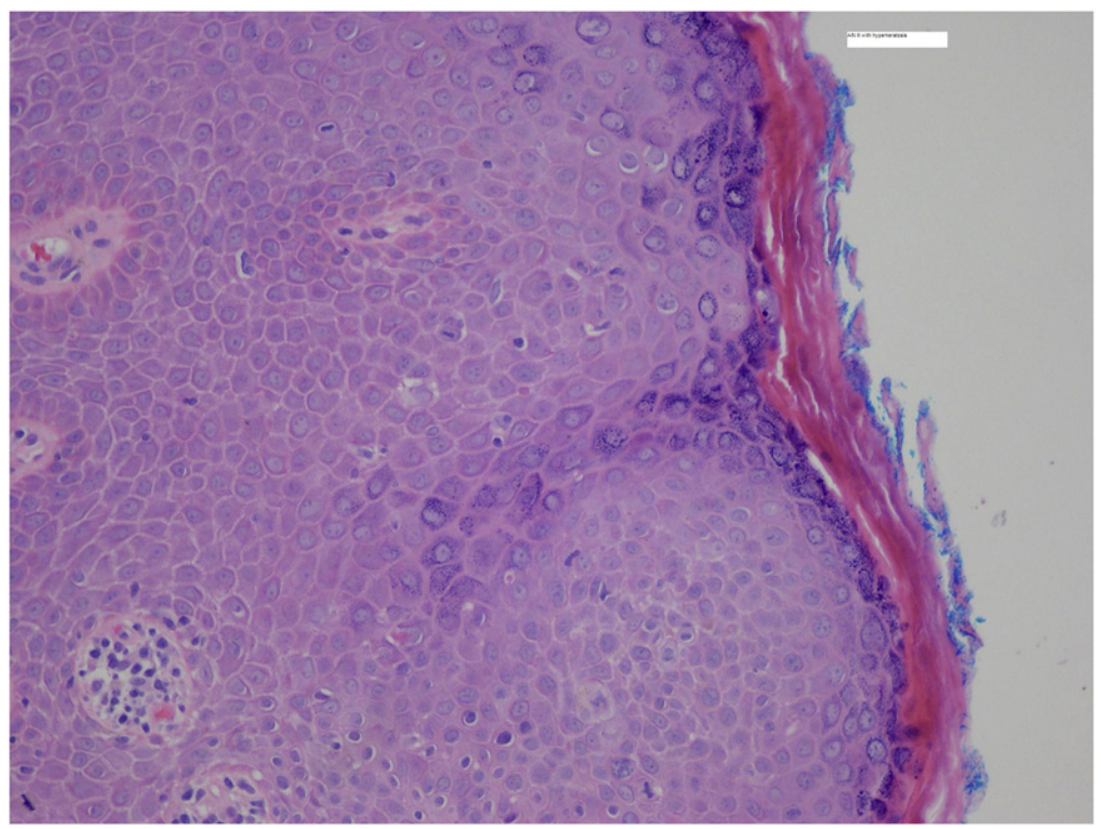

Fig. 2. Anal biopsy lesion with extensive hyperkeratosis.

to the cervix, with its easily visualized transformation zone and mucus covering, it becomes clear that AIN is more similar to VIN.

Similar to prior studies of the vulva, it is difficult to remove cells from a dry, keratinized surface [10]. The much larger and irregular surface area of the anus contributes to sampling error when compared to the easily visualized transformation zone of the cervix. Furthermore, the location of dysplasia is often found perianal and thus not amenable to detection by anal cytology [11]. Anoscopy of the perianal region allows for greater detection of AIN. Although the perianal lesions may spread into the anal canal, anal canal lesions without evidence of perianal involvement are very unusual [11].

A key discrepancy between this study and that of Palefsky et al. is the use of ThinPrep rather than glass slide [6]. Due to the dry, keratotic surface and lack of mucus, direct smears become more unreliable due to air drying. The ThinPrep allows for improved fixation and preservation. Secondly, not all patients were biopsied in the aforementioned study. Reasons for this included subject refusal and medical contraindications such as recent intake of acetylsalicylic acid, neutropenia $(<1000$ cells/ $\left.\mathrm{mm}^{3}\right)$, thrombocytopenia $\left(<75,000\right.$ platelets $\left./ \mathrm{mm}^{3}\right)$, or concurrent bacterial or viral infection in or around the anus. This study included all patients with genital intraepithelial neoplasia, save those who declined biopsy, and thus excluded.

This study demonstrated the high incidence of keratosis in anal biopsies. This keratotic covering may be the cause of anal cytology's poor sensitivity (9.4\%) in detecting AIN; as demonstrated in the cohort of 58 patients with normal anal cytology but AIN on anoscopydirected biopsy. All of which covered with keratosis. Conversely, of the patients with abnormal anal cytology and abnormal histology,

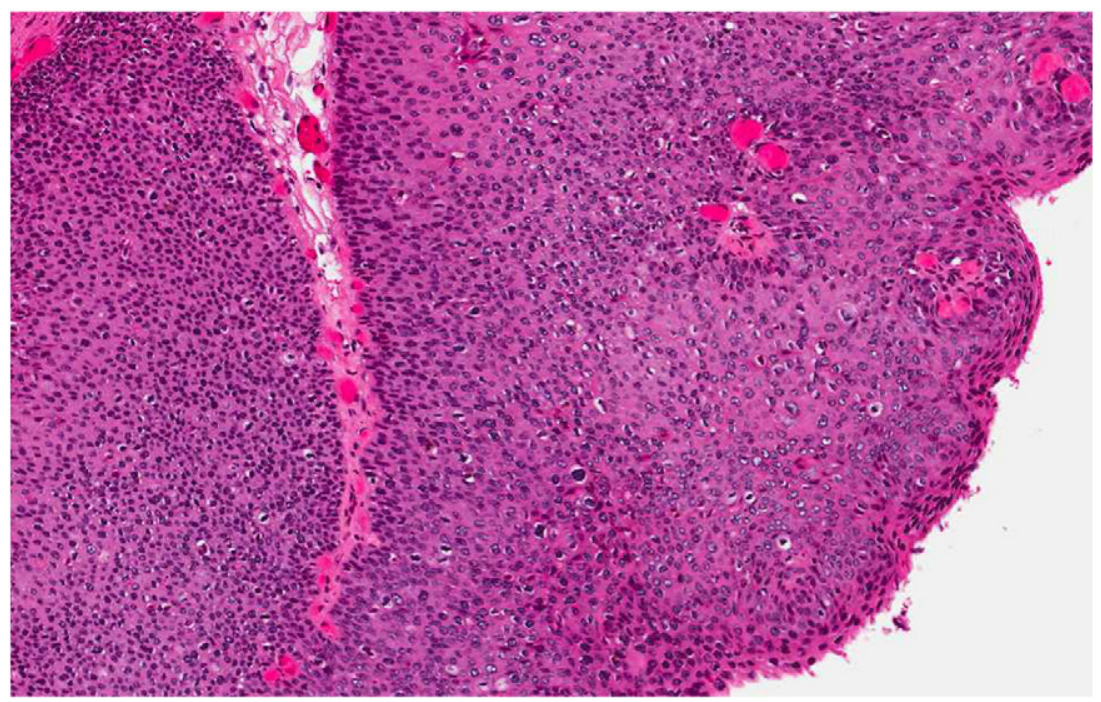

Fig. 3. Left perianal biopsy at $10 \times$ magnification with minimal keratosis. 


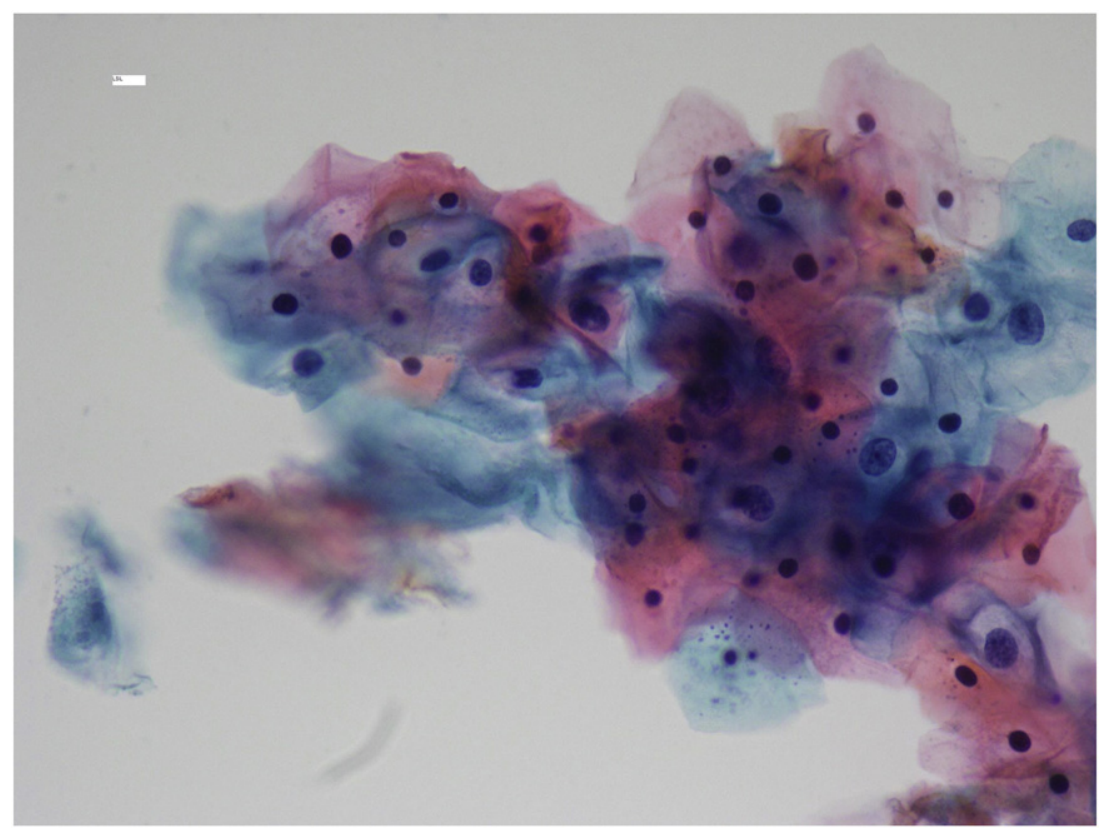

Fig. 4. Low grade squamous intraepithelial lesion from anal cytology.

all 6 biopsy specimens revealed no keratosis. Though specificity was $88.6 \%$, the FPR of $57 \%$ would let to unnecessary follow-up visits, additional exams, and unnecessary surveillance.

Furthermore, anal cytological sampling did not correlate with biopsyconfirmed diagnosis of AIN ( $\kappa$ statistic $=-0.0213$ ). Our findings do not support the use of the anal pap smear in the clinical management of AIN.

\section{Conflict of interest statement}

The authors declare that there are no conflicts of interest.

\section{References}

[1] Howlader N, N.A., Krapcho M, Neyman N, Aminou R, Waldron W, et al. SEER Cancer Statistics Review; 2011. p. 1975-2008.

[2] Benson CA, K.J., Masur H, Pau A, Holmes KK. Treating opportunistic infections among HIV-infected adults and adolescents: recommendations from CDC, the National Institutes of Health, and the HIV Medicine Association/Infectious Diseases Society of America. Morbidity and Mortality Weekly Report; 2004.
[3] Ortoski RA, Kell CS. Anal cancer and screening guidelines for human papillomavirus in men. J Am Osteopath Assoc 2011;111(3 Suppl 2):S35-43.

[4] Santoso JT, et al. Anal intraepithelial neoplasia in women with genital intraepithelial neoplasia. Obstet Gynecol 2010;116(3):578-82.

[5] Saleem AM, et al. Risk of anal cancer in a cohort with human papillomavirusrelated gynecologic neoplasm. Obstet Gynecol 2011;117(3):643-9.

[6] Palefsky JM, et al. Anal cytology as a screening tool for anal squamous intraepithelial lesions. J Acquir Immune Defic Syndr Hum Retrovirol 1997;14(5): 415-22.

[7] Lee EQ Goldstone SE. Predictors of anal dysplasia in men who have sex with men with benign cytology. Dis Colon Rectum 2011;54(3):347-51.

[8] AbdullGaffar B, et al. Lubricant, mucus, and other contaminant materials as a potential source of interpretation errors in ThinPrep cervical cytology. J Low Genit Tract Dis 2010;14(1):22-8.

[9] Holton T, et al. The effect of lubricant contamination on ThinPrep (Cytyc) cervical cytology liquid-based preparations. Cytopathology 2008;19(4):236-43.

[10] Bae-Jump VL, Bauer M, Van Le L. Cytological evaluation correlates poorly with histological diagnosis of vulvar neoplasias. J Low Genit Tract Dis 2007;11(1): 8-11.

[11] Scholefield JH, Harris D, Radcliffe A. Guidelines for management of anal intraepithelial neoplasia. Colorectal Dis 2011;13(Suppl 1):3-10. 